\title{
Scattering from a PEC Slightly Rough Surface in Chiral Media
}

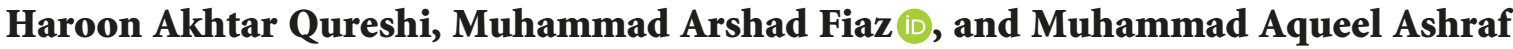 \\ Department of Electronics, Quaid-i-Azam University, Islamabad 45320, Pakistan \\ Correspondence should be addressed to Muhammad Arshad Fiaz; urarshad@gmail.com
}

Received 29 May 2017; Revised 30 November 2017; Accepted 6 December 2017; Published 20 March 2018

Academic Editor: Frederic Dumur

Copyright (C) 2018 Haroon Akhtar Qureshi et al. This is an open access article distributed under the Creative Commons Attribution License, which permits unrestricted use, distribution, and reproduction in any medium, provided the original work is properly cited.

\begin{abstract}
The scattering of left circularly polarized wave from a perfectly electric conducting (PEC) rough surface in isotropic chiral media is investigated. Since a slightly rough interface is assumed, the solution is obtained using perturbation method. Zerothorder term corresponds to solution for a flat interface which helps in making a comparison with the results reported in the literature. First-order term gives the contribution from the surface perturbations, and it is used to define incoherent bistatic scattering coefficients for a Gaussian rough surface. Higher order solution is obtained in a recursive manner. Numerical results are reported for different values of chirality, correlation length, and rms height of the surface. Diffraction efficiency is defined for a sinusoidal grating.
\end{abstract}

\section{Introduction}

Scattering from rough surfaces is an interdisciplinary research field which has many applications in optics, communication, and remote sensing. The simplest possible problem is an impenetrable rough interface, and the solution has widely been investigated [1]. The method of analysis can be analytical model such as perturbation methods (PMs) [2, 3], Kirchhoff approximation (KA) [4, 5], and extended boundary condition method [6].

The regions of validity of PM and KA are also well defined $[7,8]$. In general, PM is applied to the surface of small height. There can be an additional condition on small slopes. KA is applied to surfaces having larger radius of curvature compared with a wavelength. Numerical values of parameters such as rms height and correlation length of the surface vary with incident angle in defining the region of validity.

Numerical methods such as method of moments (MoM) [9], finite-difference time domain method (FDTDM) [10], and finite element method (FEM) [11] may also be selected to study rough surface scattering. They offer many advantages in terms of modeling and accuracy, but analytical methods are mostly applied to get more understanding of the scattering phenomena.

Scattering from a penetrable surface has been discussed in [12]. An application has been proposed to calculate the brightness temperature. Thermal emission from a layered medium bounded by a slightly rough interface has been calculated in [13]. Analytic height correlation function of rough surfaces derived from light scattering is reported in [14]. Recently, the scattering of torsional guided waves from Gaussian rough surfaces in pipe work has been discussed [15]. Full wave electromagnetic scattering from rough surfaces with buried inhomogeneities is presented by Duan and Moghaddam [16].

A chiral medium has widely been explored, and many applications have been proposed in chemistry, optics, elementary particle physics, and electromagnetics [17, 18]. Right- and left-hand circularly polarized waves have different refraction indices and different velocities in chiral medium. Consistency of hydrodynamic approximation for chiral media has been discussed by Avdoshkin et al. [19] Resonant absorption and amplification of circularly polarized waves in inhomogeneous chiral media is studied in [20]. Bassiri et al. [21] studied the electromagnetic wave 
propagation and radiation in chiral media. The effect of chirality on the polarization and intensity of the radiation from a dipole is discussed. Radiation from a layered chiral medium excited by an interior dipole is presented in [22]. Reflection from a chiral interface has been studied by many researchers [23-25]. In [23], the behavior of plane waves at the planar interfaces of mirror-conjugated chiral medium is explained. It is reported that the use of an imaging theory for chiral media is complicated for scattering problems in general since not only do the sources get imaged but the medium does also. Specifically, reflection from a PEC interface in chiral media has been evaluated in [24]. It has been demonstrated that such a conducting plane could be used for focusing in the strong chiral medium. Electromagnetic scattering from a perfectly conducting obstacle in a homogeneous chiral environment is discussed in [25].

In all the above cited work, the interfaces are assumed to be flat. At some frequencies, the interface can behave rough. The roughness affects the wave propagation, radiation, and scattering properties. The applications of rough surface scattering can be found in antenna theory, communication, and remote sensing. In this paper, the problem of reflection from a PEC rough interface placed in chiral medium is presented. Rayleigh hypothesis is utilized to get the scattered field. Zeroth-order solution obtained by PMs is used to make a comparison with that of a PEC flat interface in chiral media. Section 2 contains theoretical formulation, while numerical results are reported in Section 3. An $e^{-i \omega t}$ time dependence is assumed and suppressed throughout.

\section{Theoretical Formulation}

Consider a PEC rough surface placed in a chiral medium as shown in Figure 1. The chiral medium is defined by the following constitutive relations [23]:

$$
\begin{aligned}
& \mathbf{D}=\varepsilon(\mathbf{E}+\beta \nabla \times \mathbf{E}), \\
& \mathbf{B}=\mu(\mathbf{H}+\beta \nabla \times \mathbf{H}),
\end{aligned}
$$

where $\epsilon, \mu$, and $\beta$ are the permittivity, permeability, and chirality of the medium, respectively. The profile of the rough surface is defined as $z=f(x)$.

A left circularly polarized (LCP) plane wave is incident on the surface at an angle $\varphi_{i}$ with the $z$-axis. Its electric field is given by

$$
E_{i}=E_{0}\left[\widehat{e}_{y}+i\left(-\widehat{e}_{x} \frac{\alpha_{L i}}{\gamma_{1}}+\widehat{e}_{z} \frac{\kappa_{i}}{\gamma_{1}}\right)\right] e^{i\left(\kappa_{i} x+\alpha_{L i} z\right)},
$$

where $E_{0}$ is the amplitude of incident plane wave and $\gamma_{1}^{2}=$ $\alpha_{L i}^{2}+\kappa_{i}^{2}$ is the wave number.

The field scattered due to PEC rough surface can be written as

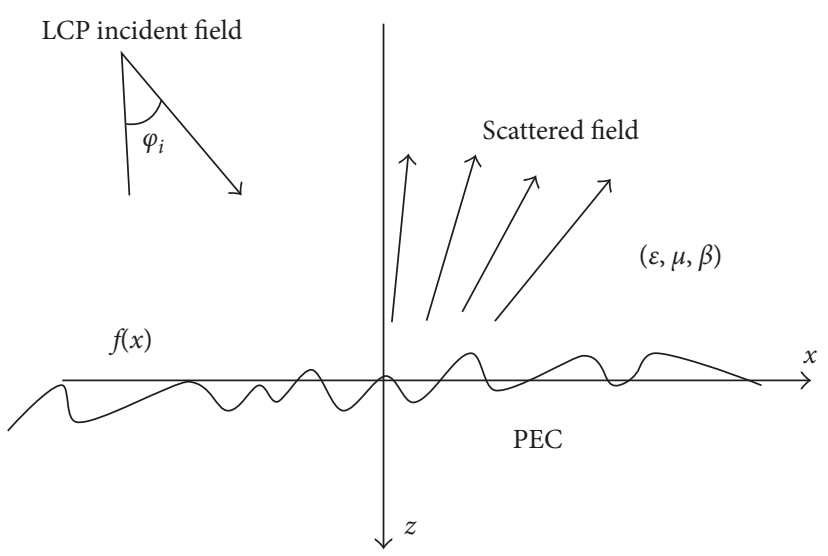

Figure 1: Scattering from a PEC rough surface in chiral media.

$$
\begin{aligned}
E_{s}= & \int_{-\infty}^{\infty}\left[\hat{e}_{y}+i\left(\hat{e}_{x} \frac{\alpha_{L}}{\gamma_{1}}+\hat{e}_{z} \frac{\kappa}{\gamma_{1}}\right)\right] R_{L}(\kappa) e^{i\left(\kappa x-\alpha_{L} z\right)} d \kappa \\
& +a_{r} \int_{-\infty}^{\infty}\left[\hat{e}_{y}-i\left(\hat{e}_{x} \frac{\alpha_{R}}{\gamma_{2}}+\hat{e}_{z} \frac{\kappa}{\gamma_{2}}\right)\right] R_{R}(\kappa) e^{i\left(\kappa x-\alpha_{R} z\right)} d \kappa,
\end{aligned}
$$

where $R_{L}(\kappa)$ and $R_{R}(\kappa)$ are the unknown coefficients for LCP and RCP scattered fields, respectively, and $a_{r}=-i \sqrt{\mu / \varepsilon}$.

The perturbation series of unknown coefficients in spectral domain is

$$
\begin{aligned}
& R_{L}(\kappa)=\sum_{m=0}^{\infty} R_{L}^{m}(\kappa), \\
& R_{R}(\kappa)=\sum_{m=0}^{\infty} R_{R}^{m}(\kappa) .
\end{aligned}
$$

It is assumed that the following conditions are satisfied:

$$
\begin{gathered}
\left|\alpha_{L i} f(x)\right| \ll 1, \\
\left|\frac{\partial f(x)}{\partial x}\right| \ll 1 .
\end{gathered}
$$

Using the power series for exponentials, the scattered field can be written as

$$
\begin{aligned}
E_{s}= & \int_{-\infty}^{\infty} \sum_{m=0}^{\infty} R_{L}^{m}(\kappa)\left(\widehat{e}_{y}+i\left(\widehat{e}_{x} \frac{\alpha_{L}}{\gamma_{1}}+\widehat{e}_{z} \frac{\kappa}{\gamma_{1}}\right)\right) e^{i \kappa x} \sum_{q=0}^{\infty} \frac{\left(-i \alpha_{L} z\right)^{q}}{q !} d \kappa \\
& +a_{r} \int_{-\infty}^{\infty} \sum_{n=0}^{\infty} R_{R}^{m}(\kappa)\left(\widehat{e}_{y}-i\left(\widehat{e}_{x} \frac{\alpha_{R}}{\gamma_{2}}+\widehat{e}_{z} \frac{\kappa}{\gamma_{2}}\right)\right) e^{i \kappa x} \sum_{q=0}^{\infty} \frac{\left(-i \alpha_{R} z\right)^{q}}{q !} d \kappa .
\end{aligned}
$$

The boundary condition is given by 


$$
\left(\widehat{e}_{z}+\frac{\partial f}{\partial x} \widehat{e}_{x}\right) \times\left(E_{i}+E_{s}\right)=0 .
$$

Applying the above boundary condition at $z=f(x)$,

$$
\begin{aligned}
& E_{0} e^{i \kappa_{i} x} \sum_{q=0}^{\infty} \frac{\left(i \alpha_{L i} f(x)\right)^{q}}{q !}+\int_{-\infty}^{\infty} e^{i \kappa x} \sum_{q=0}^{\infty} \sum_{m=0}^{\infty} R_{L}^{m}(\kappa) \frac{\left(-i \alpha_{L} f(x)\right)^{q}}{q !} d \kappa \\
& +a_{r} \int_{-\infty}^{\infty} e^{i \kappa x} \sum_{q=0}^{\infty} \sum_{m=0}^{\infty} R_{R}^{m}(\kappa) \frac{\left(-i \alpha_{R} f(x)\right)^{q}}{q !} d \kappa=0, \\
& -E_{0} i\left(\frac{\alpha_{L i}}{\gamma_{1}}+\frac{\kappa_{i}}{\gamma_{1}} \frac{\partial f}{\partial x}\right) e^{i \kappa_{i} x} \sum_{q=0}^{\infty} \frac{\left(i \alpha_{L i} z\right)^{q}}{q !} \\
& +\int_{-\infty}^{\infty} i\left(\frac{\alpha_{L}}{\gamma_{1}}-\frac{\kappa}{\gamma_{1}} \frac{\partial f}{\partial x}\right) e^{i \kappa x} \sum_{q=0}^{\infty} \sum_{m=0}^{\infty} R_{L}^{m}(\kappa) \frac{\left(-i \alpha_{L} f(x)\right)^{q}}{q !} d \kappa \\
& -a_{r} \int_{-\infty}^{\infty} i\left(\frac{\alpha_{R}}{\gamma_{2}}-\frac{\kappa}{\gamma_{2}} \frac{\partial f}{\partial x}\right) e^{i \kappa x} \sum_{q=0}^{\infty} \sum_{m=0}^{\infty} R_{R}^{m}(\kappa) \frac{\left(-i \alpha_{R} f(x)\right)^{q}}{q !} d \kappa=0 .
\end{aligned}
$$

The above infinite series is solved for unknown coefficients. The zeroth-order terms of both the above equations are

$$
\begin{aligned}
& E_{0} e^{i \kappa_{i} x}+\int_{-\infty}^{\infty} e^{i \kappa x} R_{L}^{0}(\kappa) d \kappa+a_{r} \int_{-\infty}^{\infty} e^{i \kappa x} R_{R}^{0}(\kappa) d \kappa=0 \\
& -E_{0} i \frac{\alpha_{L i}}{\gamma_{1}} e^{i \kappa_{i} x}+\int_{-\infty}^{\infty} i \frac{\alpha_{L}}{\gamma_{1}} e^{i \kappa x} R_{L}^{0}(\kappa) d \kappa \\
& -a_{r} \int_{-\infty}^{\infty} i \frac{\alpha_{R}}{\gamma_{2}} e^{i \kappa x} R_{R}^{0}(\kappa) d \kappa=0
\end{aligned}
$$

Taking the Fourier transform of the above equations gives

$$
\begin{array}{r}
E_{0} \delta\left(\kappa-\kappa_{i}\right)+R_{L}^{0}(\kappa)+a_{r} R_{R}^{0}(\kappa)=0, \\
-E_{0} \frac{\alpha_{L i}}{\gamma_{1}} \delta\left(\kappa-\kappa_{i}\right)+\frac{\alpha_{L}}{\gamma_{1}} R_{L}^{0}(\kappa)-a_{r} \frac{\alpha_{R}}{\gamma_{2}} R_{R}^{0}(\kappa)=0 .
\end{array}
$$

Solving the above equations, zero-order coefficients are obtained:

$$
\begin{aligned}
& R_{L}^{0}(\kappa)=-\frac{1}{Q_{n}}\left(\gamma_{2} \alpha_{L i}-\gamma_{1} \alpha_{R}\right) E_{0} \delta\left(\kappa-\kappa_{i}\right), \\
& R_{R}^{0}(\kappa)=\frac{1}{Q_{n}} \gamma_{2}\left(\alpha_{L i}+\alpha_{L}\right) a_{l} E_{0} \delta\left(\kappa-\kappa_{i}\right),
\end{aligned}
$$

where $Q_{n}=\gamma_{1} \alpha_{R}+\gamma_{2} \alpha_{L}$ and $a_{l}=-1 / a_{r}$.

Putting in (3), zeroth-order scattered field is obtained:

$$
\begin{aligned}
E_{s}= & -\left[\widehat{e}_{y}+i \widehat{e}_{x} \frac{\alpha_{L}}{\gamma_{1}}+i \widehat{e}_{z} \frac{\kappa}{\gamma_{1}}\right] \frac{\gamma_{2} \alpha_{L i}-\gamma_{1} \alpha_{R i}}{\gamma_{1} \alpha_{R i}+\gamma_{2} \alpha_{L i}} E_{0} e^{i\left(\kappa x-\alpha_{L} z\right)} \\
& +a_{r}\left[\widehat{e}_{y}-i \widehat{e}_{x} \frac{\alpha_{R}}{\gamma_{2}}-i \widehat{e}_{z} \frac{\kappa}{\gamma_{2}}\right] \cdot \frac{2 \gamma_{2} \alpha_{L i}}{\gamma_{1} \alpha_{R i}+\gamma_{2} \alpha_{L i}} a_{l} E_{0} e^{i\left(\kappa x-\alpha_{R} z\right)} .
\end{aligned}
$$

It corresponds to solution for a flat interface in chiral media, and the expressions are in agreement with those reported in [23]. Balancing to the first order gives

$$
\begin{aligned}
& i E_{0} \alpha_{L i} f(x) e^{i \kappa_{i} x}+\int_{-\infty}^{\infty} R_{L}^{1}(\kappa) e^{i \kappa x} d \kappa-\int_{-\infty}^{\infty} i \alpha_{L} f(x) R_{L}^{0}(\kappa) e^{i \kappa x} d \kappa \\
& +a_{r} \int_{-\infty}^{\infty} R_{R}^{1}(\kappa) e^{i \kappa x} d \kappa-a_{r} \int_{-\infty}^{\infty} i \alpha_{R} f(x) R_{R}^{0}(\kappa) e^{i \kappa x} d \kappa=0, \\
& E_{0} \frac{\alpha_{L i}^{2}}{\gamma_{1}} f(x) e^{i \kappa x}-i E_{0} \frac{\kappa_{i}}{\gamma_{1}} \frac{\partial f}{\partial x} e^{i \kappa_{i} x}+\int_{-\infty}^{\infty} i \frac{\alpha_{L}}{\gamma_{1}} R_{L}^{1}(\kappa) e^{i \kappa x} d \kappa \\
& +\int_{-\infty}^{\infty} \frac{\alpha_{L}^{2}}{\gamma_{1}} f(x) R_{L}^{0}(\kappa) e^{i \kappa x} d \kappa-\int_{-\infty}^{\infty} i \frac{\kappa}{\gamma_{1}} \frac{\partial f}{\partial x} R_{L}^{0}(\kappa) e^{i \kappa x} d \kappa \\
& -a_{r} \int_{-\infty}^{\infty} i \frac{\alpha_{R}}{\gamma_{2}} R_{R}^{1}(\kappa) e^{i \kappa x} d \kappa-a_{r} \int_{-\infty}^{\infty} \frac{\alpha_{R}^{2}}{\gamma_{2}} f(x) R_{R}^{0}(\kappa) e^{i \kappa x} d \kappa \\
& +a_{r} \int_{-\infty}^{\infty} i \frac{\kappa}{\gamma_{2}} \frac{\partial f}{\partial x} R_{R}^{0}(\kappa) e^{i \kappa x} d \kappa=0 .
\end{aligned}
$$

Taking the Fourier transfer of the above equations,

$$
R_{L}^{1}(\kappa)+a_{r} R_{R}^{1}(\kappa)=\left[\alpha_{L i} C+a_{r} \alpha_{R i} D-E_{0} \alpha_{L i}\right] i F\left(\kappa-\kappa_{i}\right),
$$

$$
\begin{aligned}
& \frac{\alpha_{L}}{\gamma_{1}} R_{L}^{1}(\kappa)-a_{r} \frac{\alpha_{R}}{\gamma_{2}} R_{R}^{1}(\kappa)=\left[\frac{E_{0}}{\gamma_{1}}\left(\kappa \kappa_{i}-\kappa_{i}^{2}-\alpha_{L i}^{2}\right)\right. \\
& \left.+\frac{1}{\gamma_{1}}\left(\alpha_{L i}^{2}+\kappa \kappa_{i}-\kappa_{i}^{2}\right) C-\frac{a_{r}}{\gamma_{2}}\left(\alpha_{R i}^{2}+\kappa \kappa_{i}-\kappa_{i}^{2}\right) D\right] i F\left(\kappa-\kappa_{i}\right),
\end{aligned}
$$

where

$$
\begin{aligned}
& C=\frac{\gamma_{2} \alpha_{L i}-\gamma_{1} \alpha_{R i}}{\gamma_{1} \alpha_{R i}+\gamma_{2} \alpha_{L i}} E_{0}, \\
& D=\frac{2 \gamma_{2} \alpha_{L i}}{\gamma_{1} \alpha_{R i}+\gamma_{2} \alpha_{L i}} a_{l} E_{0} .
\end{aligned}
$$

From the above equations, it can be noted that the firstorder solution can be written in terms of zeroth-order solution. Solving the above equations, first-order coefficients may be written as

$$
\begin{aligned}
R_{L}^{1}(\kappa)= & \frac{i}{Q_{n}}\left[E_{0}\left[\gamma_{2}\left(\kappa \kappa_{i}-\kappa_{i}^{2}-\alpha_{L i}^{2}\right)-\alpha_{R} \alpha_{L i} \gamma_{1}\right]\right. \\
& +\left[\gamma_{2}\left(\alpha_{L i}^{2}+\kappa \kappa_{i}-\kappa_{i}^{2}\right)+\gamma_{1} \alpha_{R} \alpha_{L i}\right] C \\
& \left.-\gamma_{1}\left[\alpha_{R i}^{2}+\kappa \kappa_{i}-\kappa_{i}^{2}-\alpha_{R} \alpha_{R i}\right] a_{r} D\right] F\left(\kappa-\kappa_{i}\right), \\
R_{R}^{1}(\kappa)= & \frac{i}{a_{r} Q_{n}}\left[E_{0}\left[\gamma_{2}\left(\kappa \kappa_{i}-\alpha_{L i}^{2}-\kappa_{i}^{2}\right)-\gamma_{2} \alpha_{L} \alpha_{L i}\right]\right. \\
& +\left[\gamma_{2}\left[\alpha_{L i}^{2}+\kappa \kappa_{i}-\kappa_{i}^{2}+\alpha_{L} \alpha_{L i}\right]\right] C \\
& \left.-\left[\gamma_{1}\left(\alpha_{R i}^{2}+\kappa \kappa_{i}-\kappa_{i}^{2}\right)-\gamma_{2} \alpha_{L} \alpha_{R i}\right] D\right] F\left(\kappa-\kappa_{i}\right) .
\end{aligned}
$$




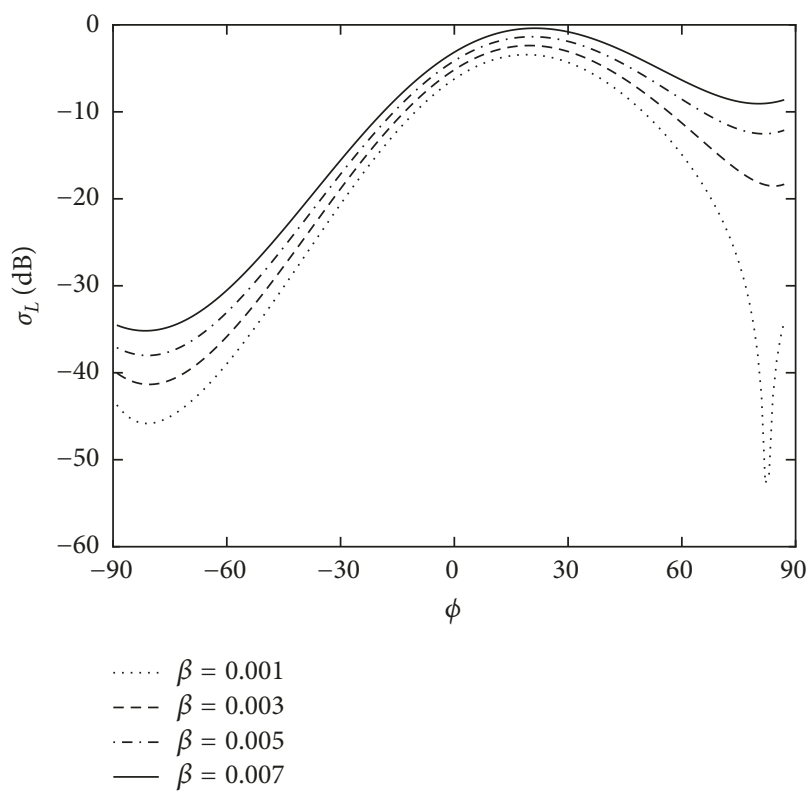

(a)

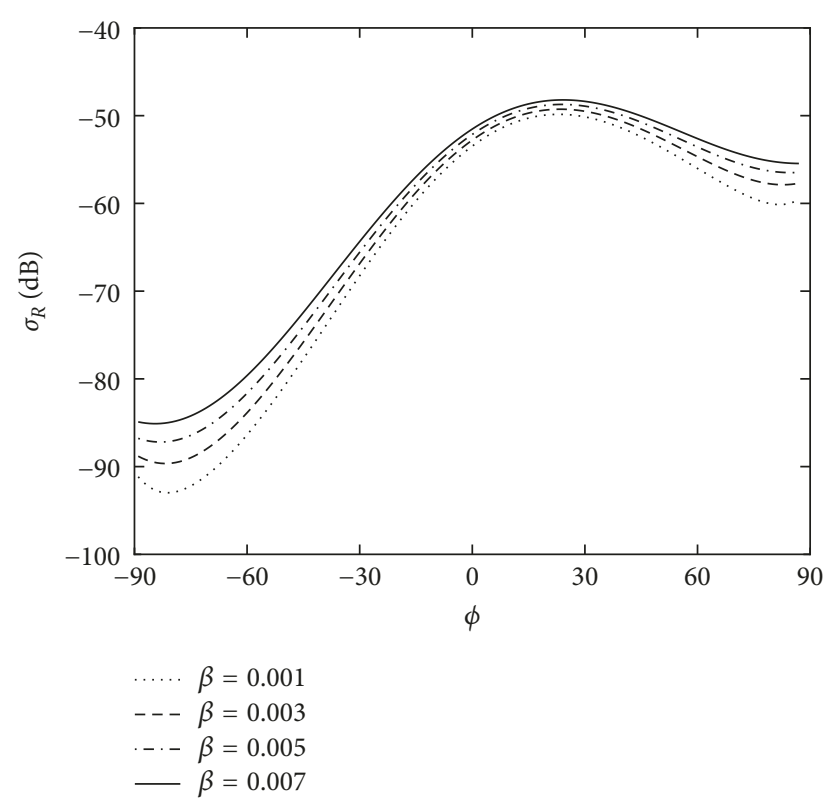

(b)

FIGURE 2: LCP and RCP scattering coefficients for different values of chirality, where $\varphi_{i}=30^{\circ}, \varepsilon_{r}=4, h=0.05$, and $l_{c}=0.35$.

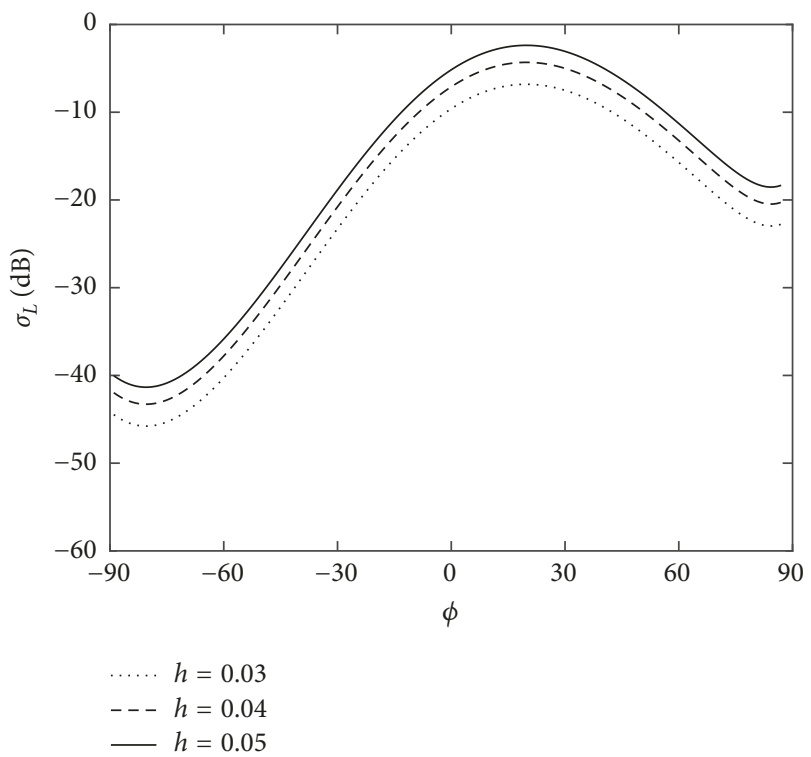

(a)

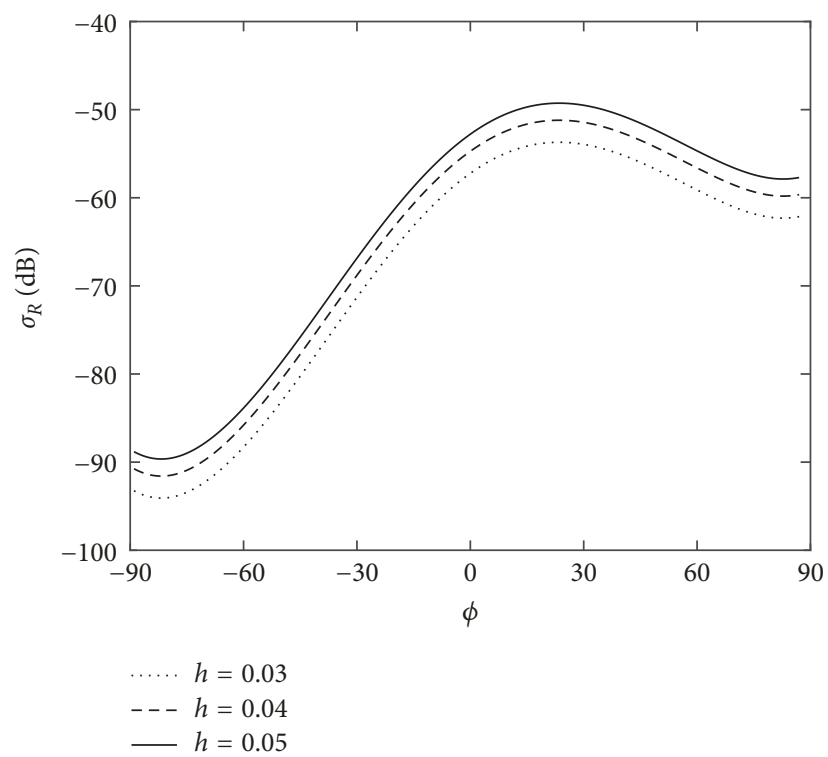

(b)

FiguRE 3: LCP and RCP scattering coefficients for different values of rms height, where $\varphi_{i}=30^{\circ}, \varepsilon_{r}=4, \beta=0.03$, and $l_{c}=0.35$.

Finally, the solution up to any order can be found via recursive computation.

\section{Numerical Analysis}

Numerical implementation of the theoretical formulation is done in this section. A rough surface profile has to be selected, and its Fourier transform is calculated. Two cases have been considered here: one is a sinusoidal surface and the other is a Gaussian rough surface. First, consider a sinusoidal surface defined as

$$
f(x)=A \cos \left(\frac{2 \pi}{\lambda_{s}} x\right),
$$

where $A$ and $\lambda_{s}$ are the amplitude and period of the surface, respectively. Using the Fourier transform of sinusoidal surface, the scattered field can be written as 


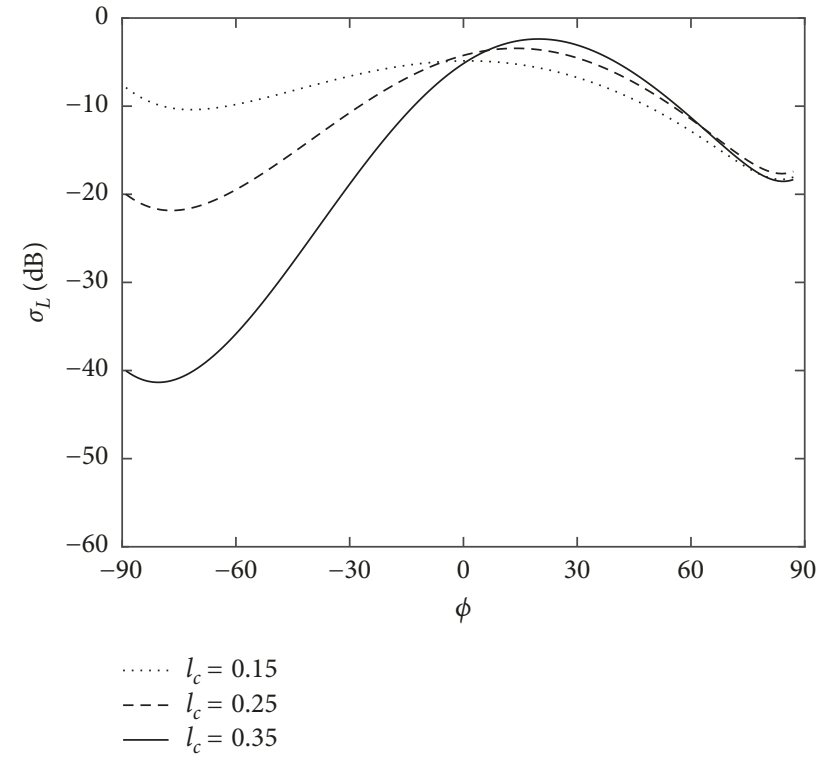

(a)

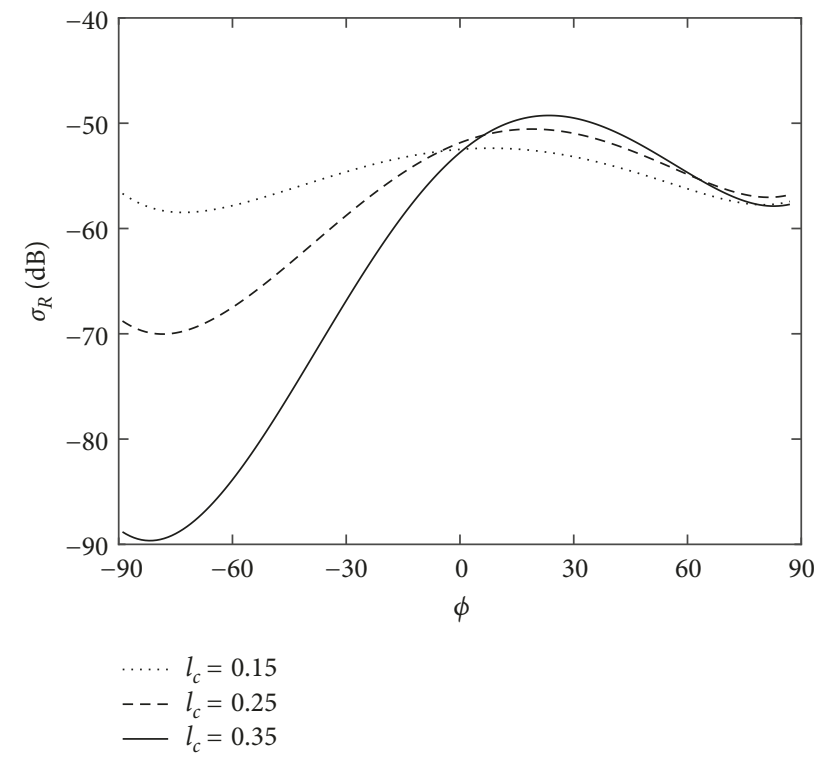

(b)

FIGURE 4: LCP and RCP scattering coefficients for different values of correlation length, where $\varphi_{i}=30^{\circ}, \varepsilon_{r}=4, \beta=0.03$, and $h=0.05$.

$$
E_{s}=\sum_{p=-1}^{1} R_{L}^{\sin }\left(\kappa_{p x}\right) e^{i\left(\kappa_{p x} x-\alpha_{L p z} z\right)}
$$

where

$$
\begin{aligned}
R_{L}^{\sin }\left(\kappa_{p x}\right)= & \frac{\gamma_{2} \alpha_{L i}-\gamma_{1} \alpha_{R i}}{\gamma_{1} \alpha_{R i}+\gamma_{2} \alpha_{L i}} E_{0}, \quad p=0 \\
R_{L}^{\sin }\left(\kappa_{p x}\right)= & \frac{i}{\gamma_{1} \alpha_{R p z}+\gamma_{2} \alpha_{L p z}} \times\left\{\left[\gamma_{2}\left(\kappa_{p x} \kappa_{i}-\kappa_{i}^{2}-\alpha_{L i}^{2}\right)\right.\right. \\
& \left.-\alpha_{R p z} \alpha_{L i} \gamma_{1}\right] E_{0}+\left[\gamma_{2}\left(\alpha_{L i}^{2}+\kappa_{p x} \kappa_{i}-\kappa_{i}^{2}\right)\right. \\
& \left.+\gamma_{1} \alpha_{R p z} \alpha_{L i}\right] C-\gamma_{1}\left[\alpha_{R i}^{2}+\kappa_{p x} \kappa_{i}-\kappa_{i}^{2}\right. \\
& \left.\left.-\alpha_{R p z} \alpha_{R i}\right] a_{r} D\right\}, \quad p= \pm 1, \\
\kappa_{p x}= & \kappa_{i}+\frac{2 \pi p}{\lambda_{s}}, \\
\alpha_{L p z}= & \sqrt{\gamma_{1}^{2}-\kappa_{p x}^{2}}, \\
\alpha_{R p z}= & \sqrt{\gamma_{2}^{2}-\kappa_{p x}^{2}} .
\end{aligned}
$$

In a similar way, the expressions for RCP scattered field can be written. The $p$ th order diffraction efficiency $\eta_{p}$ is defined as the fraction of the incident energy which is reflected in the $\kappa_{p x}$ direction, and it is given by

$$
\eta_{p}^{L}=\frac{\operatorname{Re}\left(\alpha_{L p z}\right)}{\alpha_{L i}}\left|R_{L}^{\sin }\left(\kappa_{p x}\right)\right|^{2} .
$$

Now, consider a rough surface with Gaussian roughness spectrum. The power spectral density $W\left(k_{x}\right)$ is given by

$$
W\left(k_{x}\right)=\frac{h^{2} l_{c}}{2 \sqrt{\pi}} e^{-\left(\kappa^{2} l_{c}^{2} / 4\right)},
$$

where $l_{c}$ is the correlation length and $h$ is the root mean square (rms) height of the rough surface. The rms slope is given by [1]

$$
s=\frac{\sqrt{2} h}{l_{c}}
$$

Since both LCP and RCP waves are scattered for LCP incidence, the LCP and RCP incoherent bistatic scattering coefficients can be expressed as

$$
\begin{aligned}
& \sigma_{L}(\kappa)=\frac{\alpha_{L}}{\alpha_{L i}} W\left(\kappa-\kappa_{i}\right)\left[\gamma_{L}\right]^{2}, \\
& \sigma_{R}(\kappa)=\frac{\alpha_{R}}{\alpha_{L i}} W\left(\kappa-\kappa_{i}\right)\left[\gamma_{R}\right]^{2},
\end{aligned}
$$

where

$$
\begin{aligned}
\gamma_{L}= & \frac{i}{Q_{n}}\left\{\left[\gamma_{2}\left(\kappa \kappa_{i}-\kappa_{i}^{2}-\alpha_{L i}^{2}\right)-\alpha_{R} \alpha_{L i} \gamma_{1}\right] E_{0}\right. \\
& +\left[\gamma_{2}\left(\alpha_{L i}^{2}+\kappa \kappa_{i}-\kappa_{i}^{2}\right)+\gamma_{1} \alpha_{R} \alpha_{L i}\right] C \\
& \left.-\gamma_{1}\left[\alpha_{R i}^{2}+\kappa \kappa_{i}-\kappa_{i}^{2}-\alpha_{R} \alpha_{R i}\right] a_{r} D\right\}, \\
\gamma_{R}= & \frac{i}{a_{r} Q_{n}}\left\{\left[\gamma_{2}\left(\kappa \kappa_{i}-\alpha_{L i}^{2}-\kappa_{i}^{2}\right)-\gamma_{2} \alpha_{L} \alpha_{L i}\right] E_{0}\right. \\
& +\left[\gamma_{2}\left[\alpha_{L i}^{2}+\kappa \kappa_{i}-\kappa_{i}^{2}+\alpha_{L} \alpha_{L i}\right]\right] C \\
& \left.-\left[\gamma_{1}\left(\alpha_{R i}^{2}+\kappa \kappa_{i}-\kappa_{i}^{2}\right)-\gamma_{2} \alpha_{L} \alpha_{R i}\right] D\right\} .
\end{aligned}
$$


Figure 2 shows the incoherent bistatic coefficients for different values of chirality parameter. It can be observed that RCP scattered field is smaller than LCP scattered field. Moreover, scattered field increases as the chirality increases. Chirality may change the angular behavior significantly at some scattered angles as described by the minima in Figure 2(a). Figure 3 shows the scattering pattern for the rms height. The smaller $h$ corresponds to a flat interface. As expected, the scattered field increases with the increase in rms height. The angular behavior of LCP and RCP scattered fields is same. The effect on scattering pattern for different values of correlation length is shown in Figure 4. The effect of slope (correlation length) can be observed on the scattering pattern away from the specular direction. The scattered field decreases away from specular direction $\varphi=30^{\circ}$. It can also be observed that the slope has more effect on scattering behavior as compared to chirality. The numerical results show that slight roughness of the interface cannot be ignored in order to be close to the experimental results and for the design purposes.

\section{Conclusion}

Scattered field from a PEC rough surface in chiral media has been studied. PM is applied to obtain scattered field components. In general, the higher order scattered field can be obtained using lower order coefficients by utilizing the recursive nature of the problem. Two cases, sinusoidal and Gaussian rough surfaces, are considered, and the expressions of the LCP and RCP incoherent bistatic scattering coefficients have been reported. Scattering pattern is observed for chirality parameter, height, and correlation length of the Gaussian rough surface. Diffraction efficiency has been defined for a rough surface with sinusoidal profile. This analysis can also be used for a triangular grating.

\section{Conflicts of Interest}

No potential conflicts of interest are reported by the authors.

\section{References}

[1] L. Tsang, J. A. Kong, K. H Ding, and C. O. Ao, Scattering of Electromagnetic Waves: Theories and Applications, Wiley, New York, NY, USA, 2001.

[2] S. O. Rice, "Reflection of electromagnetic wave by slightly rough surfaces," Communications on Pure and Applied Mathematics, vol. 4, no. 2-3, pp. 351-378, 1951.

[3] M. Sanamzadeh, L. Tsang, J. T. Johnson, R. J. Burkholder, and S. Tan, "Scattering of electromagnetic waves from 3D multilayer random rough surfaces based on the second-order small perturbation method: energy conservation, reflectivity, and emissivity," Journal of the Optical Society of America A, vol. 34, no. 3, pp. 395-409, 2017.

[4] Z. S. Wu, J. J. Zhang, and L. Zhao, "Composite electromagnetic scattering from the plate target above a one-dimensional sea surface: taking the diffraction into account," Progress in Electromagnetics Research, vol. 92, pp. 317-331, 2009.
[5] J. Tian, J. Tong, J. Shi, and L. Gui, “A new approximate fast method of computing the scattering from multilayer rough surfaces based on the Kirchhoff approximation," Radio Science, vol. 52, no. 2, pp. 186-195, 2017.

[6] L. Guo and Z. Wu, "Application of the extended boundary condition method to electromagnetic scattering from rough dielectric fractal sea surface," Journal of Electromagnetic Waves and Applications, vol. 18, no. 9, pp. 1219-1234, 2004.

[7] E. I. Thorsos, "The validity of the Kirchhoff approximation for rough surface scattering using a Gaussian roughness spectrum," Journal of the Acoustical Society of America, vol. 83, no. 1, pp. 78-92, 1988.

[8] M. F. Chen and A. K. Fung, "A numerical study of the regions of validity of the Kirchhoff and small-perturbation rough surface scattering models," Radio Science, vol. 23, no. 2, pp. 163-170, 1988.

[9] D. Liang, P. Xu, L. Tsang, Z. Gui, and K.-S. Chen, "Electromagnetic scattering by rough surfaces with large heights and slopes with applications to microwave remote sensing of rough surface over layered media," Progress in Electromagnetics Research, vol. 95, pp. 199-218, 2009.

[10] J. Li, L. X. Guo, H. Zeng, and X. B. Han, "Message-passinginterface-based parallel FDTD investigation on the EM scattering from a 1-D rough sea surface using uniaxial perfectly matched layer absorbing boundary," Journal of the Optical Society of America A, vol. 26, no. 6, pp. 1494-1502, 2009.

[11] S. H. Lou, L. Tsang, and C. H. Chan, "Application of finite element method to Monte Carlo simulations of scattering of waves by random rough surfaces: penetrable case," Waves in Random Media, vol. 1, no. 4, pp. 287-307, 1991.

[12] M. A. Demir and J. T. Johnson, "Fourth- and higher-order small-perturbation solution for scattering from dielectric rough surfaces," Journal of the Optical Society of America A, vol. 20, no. 12, pp. 2330-2337, 2003.

[13] M. A. Demir and J. T. Johnson, "Thermal emission from a layered medium bounded by a slightly rough interface," IEEE Transactions on Geoscience and Remote Sensing, vol. 39, no. 2, pp. 368-378, 2001.

[14] M. Zamani, F. Shafiei, S. M. Fazeli, M. C. Downer, and G. R. Jafari, "Analytic height correlation function of rough surfaces derived from light scattering," Physical Review E, vol. 94, p. 042809, 2016.

[15] J. Dobsonand and P. Cawley, "The scattering of torsional guided waves from Gaussian rough surfaces in pipework," Journal of the Acoustical Society of America, vol. 141, no. 3, pp. 1852-1861, 2017.

[16] X. Duan and M. Moghaddam, "Full-Wave Electromagnetic scattering from rough surfaces with buried inhomogeneities," IEEE Transactions on Geoscience and Remote Sensing, vol. 55, no. 6, pp. 3338-3353, 2017.

[17] N. Engheta and D. L. Jaggard, "Electromagnetic chirality and its applications," IEEE Antennas and Propagation Society Newsletter, vol. 30, no. 5, pp. 6-12, 1988.

[18] N. L. Tsitsas, A. Lakhtakia, and D. J. Frantzeskakis, "Vector solitons in nonlinear isotropic chiral metamaterials," Journal of Physics A: Mathematical and Theoretical, vol. 44, no. 43, p. 435203, 2011.

[19] A. Avdoshkin, V. P. Kirilin, A. V. Sadofyev, and V. I. Zakharov, "On consistency of hydrodynamic approximation for chiral media," Physics Letters B, vol. 755, pp. 1-7, 2016.

[20] S. Kim and K. Kim, "Resonant absorption and amplification of circularly-polarized waves in inhomogeneous chiral media," Optics Express, vol. 24, no. 2, p. 255084, 2016. 
[21] S. Basiri, "Electromagnetic waves propagation and radiation in chiral media," Ph.D. thesis, California institute of technology, Pasadena, California, 1987.

[22] C. Athanasiadis and N. L. Tsitsas, "Radiation relations for electromagnetic excitation of a layered chiral medium by an interior dipole," Journal of Mathematical Physics, vol. 49, p. 013510, 2008.

[23] A. Lakhtakia, V. V. Varadan, and V. K. Varadan, "What happens to plane waves at the planar interfaces of mirrorconjugated chiral media," Journal of the Optical Society of America A, vol. 6, no. 1, pp. 23-26, 1989.

[24] C. Zhang and T. J. Cui, "Negative reflections of electromagnetic waves in chiral media," 2006, http://arxiv.org/abs/ physics/0610172.

[25] C. Athanasiadis, G. Costakis, and I. G. Stratis, "Electromagnetic scattering by a perfectly conducting obstacle in a homogeneous chiral environment: solvability and lowfrequency theory," Mathematical Methods in the Applied Sciences, vol. 25, no. 11, pp. 927-944, 2002. 


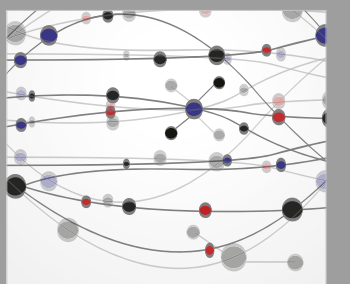

The Scientific World Journal
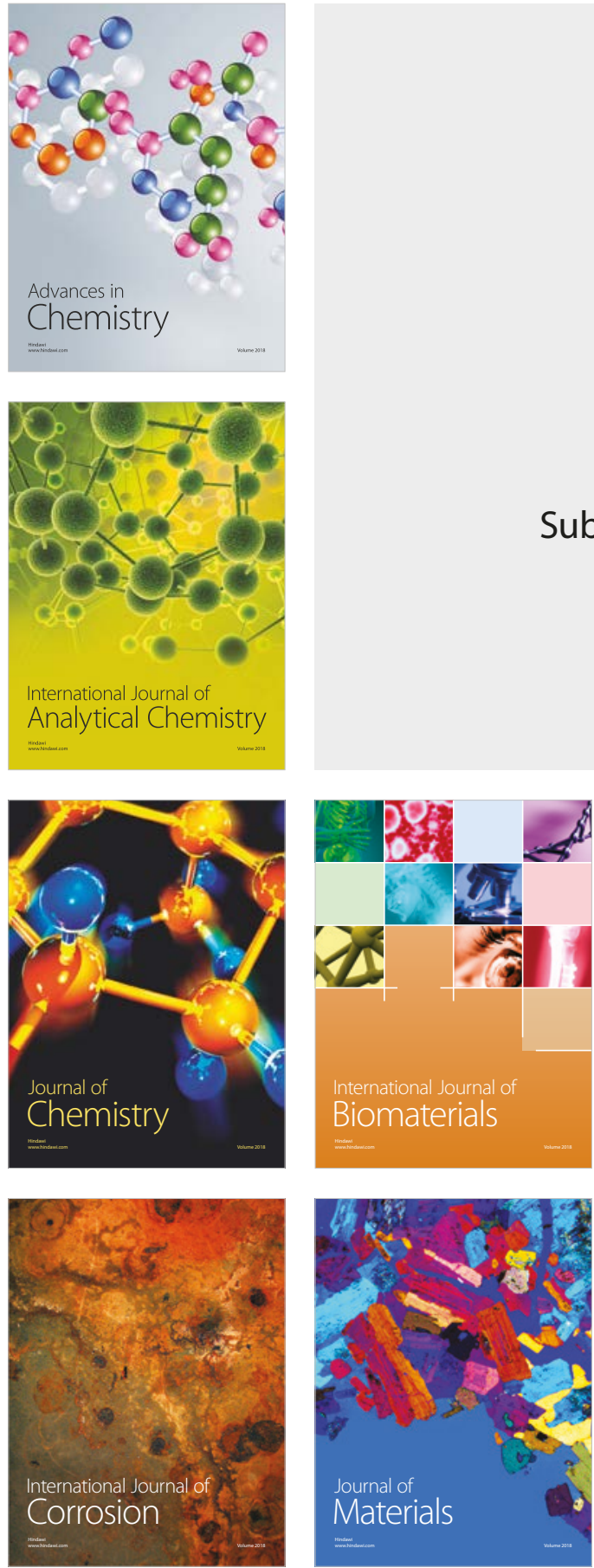

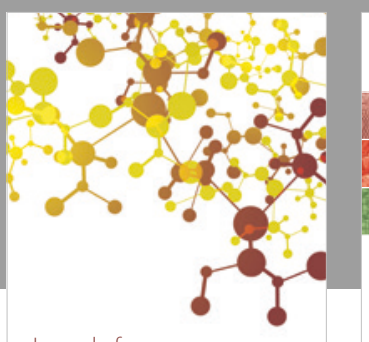

Journal of

Applied Chemistry
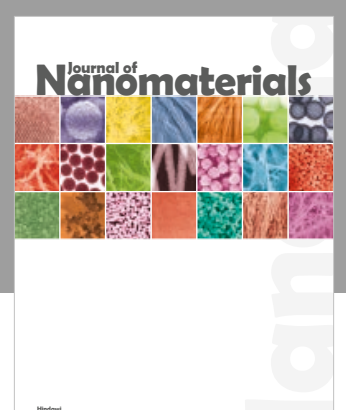

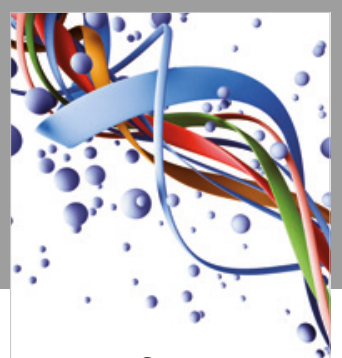

Scientifica

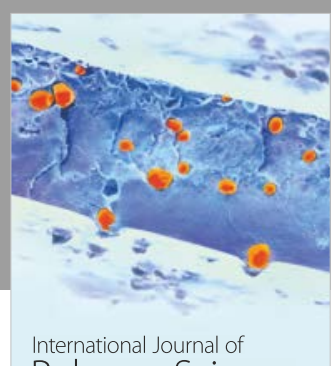

Polymer Science

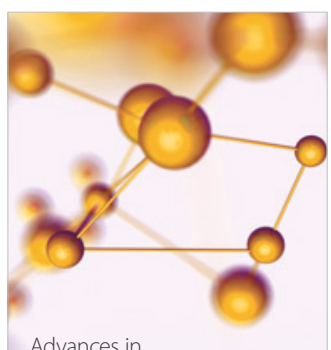

Physical Chemistry
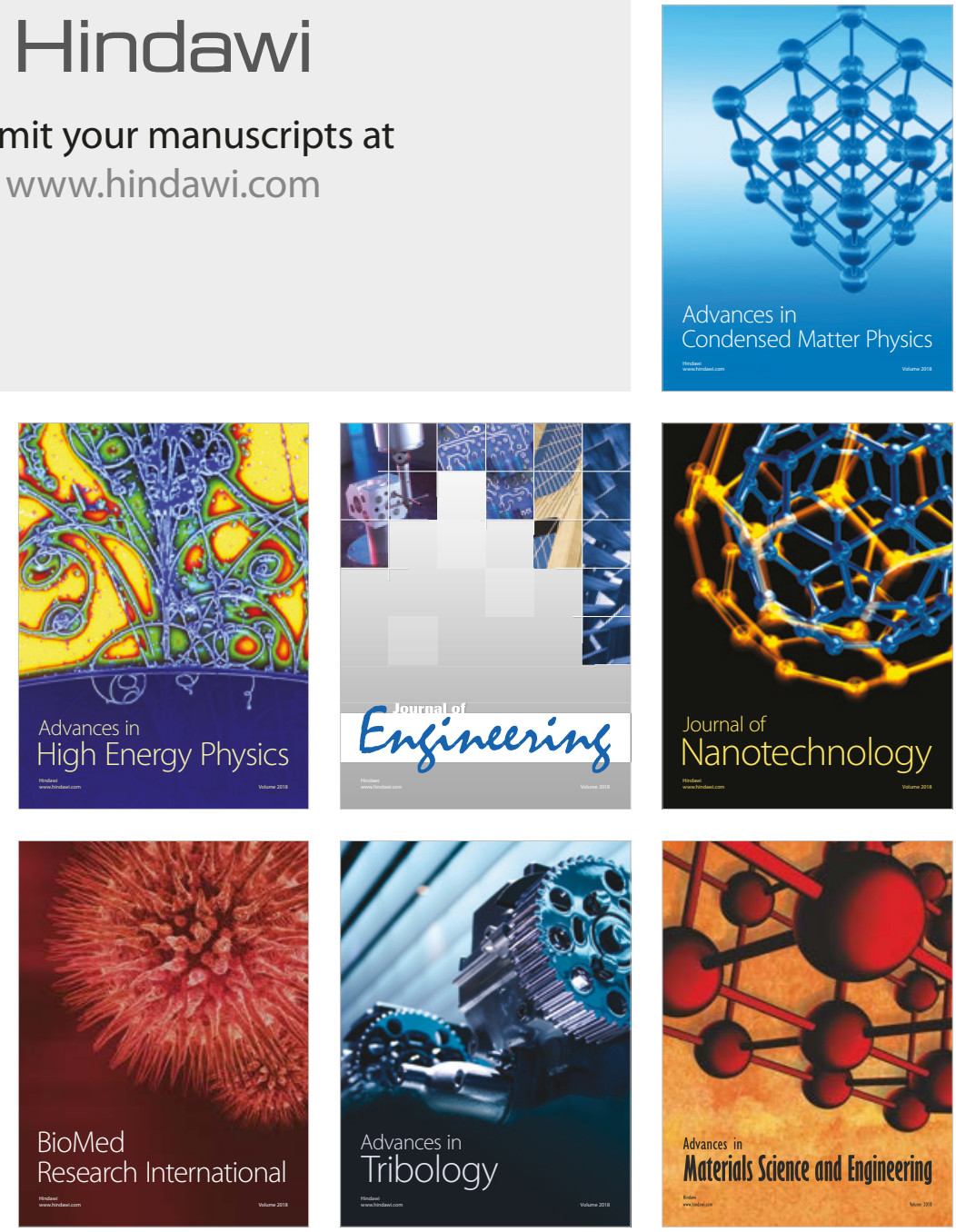